\title{
Évaluation du risque de syndrome métabolique chez les travailleurs postés en Tunisie
}

Imène Kacem, ${ }^{1,2}$ Maher Maoua, ${ }^{1,2}$ Yosra Hasni, ${ }^{1,3}$ Houda Kalboussi, ${ }^{1,2}$ Meriem Hafsia, ${ }^{1,2}$ Souha Souguir, ${ }^{1,3}$ Senda Ouerdani, ${ }^{1,3}$ Amel Maaroufi, ${ }^{1,3}$ Olfa El Maalel $^{1,2}$ et Nejib Mrizak ${ }^{1,2}$

${ }^{1}$ Faculté de Médecine Ibn Al Jazzar, Sousse (Tunisie). ${ }^{2}$ Service de Médecine du Travail et de Pathologie Professionnelle, Centre hospitalier universitaire (CHU) Farhat Hached, Sousse (Tunisie) (Correspondance à adresser à I. Kacem : Kacem.imane@live.fr). ${ }^{3}$ Service d'endocrinologie, CHU Farhat Hached, Sousse (Tunisie).

\section{Résumé}

Contexte : Le travail posté est à l'origine de problèmes de santé pour les travailleurs dans le monde entier. Le syndrome métabolique est l'une des pathologies qui peuvent survenir du fait de ce mode de travail.

Objectifs: Recherched'uneéventuelle association entreletravail postéetlerisquededévelopperun syndromemétabolique (SM).

Méthodes : il s'agit d'une étude transversale menée auprès des agents de sexe masculin d'une centrale de production d'électricité au centre de la Tunisie. Cette population était répartie en deux groupes selon l'organisation de leur travail, soit un groupe de travailleurs postés et un groupe de travailleurs non postés. Le recueil des données s'est basé sur un questionnaire, un examen clinique et un bilan biologique. La définition de la Fédération internationale du diabète 2005 a été adoptée pour retenir le diagnostic de SM.

Résultats : au total, 263 agents ont répondu à notre convocation, soit un taux de participation de $65,5 \%$. Le groupe des travailleurs postés représentait $48,3 \%$ des participants et le groupe des travailleurs non postés $51,7 \%$. Le diagnostic de SM a été retenu chez $51,2 \%$ des travailleurs postés et chez $27,2 \%$ des travailleurs non postés, avec une différence statistiquement significative $\left(\mathrm{p}<10^{-3}\right)$. Cette association a persisté après ajustement des variables de confusion avec un OR de 2,64 [1,38 - 5,04].

Conclusion : notre étude a permis d'objectiver le risque de développement du SM chez les travailleurs postés. Ces constats relèvent l'importance de la prise de mesures préventives. Cette prévention repose non seulement sur des mesures hygiéno - diététiques et une activité physique régulière mais aussi sur l'amélioration des conditions de travail.

Mots clés : travail posté, syndrome métabolique, facteurs de risque, Tunisie

Citation: Kacem I; Maoua M ; Hasni Y ; Kalboussi H ; Hafsia M ; Souguir S et al. Évaluation du risque de syndrome métabolique chez les travailleurs postés en Tunisie. East Mediterr Health J. 2019;25(10):677-685. https://doi.org/10.26719/emhj.19.040

Reçu : 16/01/18; accepté : 05/06/18

(c) Organisation mondiale de la Santé 2019. Certains droits réservés. La présente publication est disponible sous la licence Creative Commons Attribution - Pas d'utilisation commerciale - Partage dans les mêmes conditions 3.0 IGO (CC BY - NC - SA 3.0 IGO ; (https://creativecommons.org/ licenses/by-nc-sa/3.0/igo).

\section{Introduction}

Le syndrome métabolique (SM) désigne un état particulier de morbidité caractérisé par l'association de plusieurs anomalies métaboliques (1). Le SM est une pathologie grave à cause de la fréquence des complications qui lui sont associées et du risque accru de mortalité (2). Il s'agit d'une affection fréquente dont l'incidence ne cesse d'augmenter (3). En Tunisie, la prévalence globale du SM se situe aux alentours de $31,2 \%$ (4).

La prévalence accrue de cette affection a été attribuée aux changements de mode de vie, particulièrement en ce qui concerne les nouvelles habitudes alimentaires et la sédentarité (5). La vie moderne a également imposé des changements considérables dans l'environnement de travail (6). Parmi ces changements figure le travail posté, qui revêt une importance grandissante en raison du nombre de plus en plus élevé de travailleurs concernés par ce mode d'activité professionnelle (7).

Le travail posté, qui intéresse environ $20 \%$ des ouvriers dans les pays industrialisés, a fait l'objet de nombreuses études afin d'évaluer ses répercussions sur les mécanismes d'adaptation de l'organisme. En introduisant un décalage entre le rythme de travail, les prises alimentaires et les phases de repos entre le jour et la nuit, le travail posté peut avoir un impact direct sur l'horloge biologique et entraîner ainsi des perturbations dans les variations cycliques, hormonales et biologiques (8). En effet, un sommeil insuffisant ou de mauvaise qualité en rapport avec le travail en horaires décalés et un stress psychosocial en rapport avec une vie sociale bouleversée peuvent être responsables de dérégulations hormonales, notamment des hormones de l'homéostasie énergétique, d'un risque accru de gain de poids et de développement de pathologies métaboliques (9).

Plusieurs auteurs se sont intéressés à l'étude de la relation entre le travail posté et le risque de développement du SM, avec des résultats plaidant en faveur d'une relation de causalité (10). Cependant, une récente revue systématique a conclu à l'absence de preuves suffisantes pourcette relation. Les auteurs de cette revue ontégalement souligné l'importance d'une prise en considération des facteurs de risque impliqués dans la liaison de la chaîne causale complexe du travail posté et du SM (11). Ainsi, les études menées dans ce domaine ne sont pas concluantes et les résultats rapportés sont souvent contradictoires 
et d'interprétation difficile en raison de la diversité des définitions et des méthodologies adoptées (11).

C'est dans ce cadre qu'a été réalisée la présente étude dont l'objectif était de rechercher une éventuelle association entre le travail posté et le risque de développer un SM.

\section{Méthodes}

Il s'agit d'une étude transversale qui a intéressél'ensemble des agents de sexe masculin d'une centrale de production d'électricité, située dans la ville de Sousse (Tunisie). La population d'étude était composée de deux groupes selon l'organisation de leur travail. Le premier groupe posté était composé des travailleurs occupant un poste continu de rotation courte sous le mode $3 \times 8$ heures. Le second groupe non posté était composé de travailleurs à poste fixe ainsi que d'agents administratifs bénéficiant des horaires de huit heures avec un repos le samedi et le dimanche. Les critères d'inclusion étaient l'ancienneté au poste de travail de plus d'un an ainsi qu'un examen médical d'embauche et un bilan métabolique initial normaux. Ont été exclus de l'étude les salariés de sexe féminin, les sujets présentant des antécédents pathologiques d'une endocrinopathie ou d'une insuffisance rénale, ceux suivis pour diabète, hypertension artérielle (HTA) ou dyslipidémie avant l'embauche, ainsi que les salariés ayant reçu une corticothérapie au long cours.

Le recueil des données a été basé sur un questionnaire médical, un examen physique et un bilan biologique. Le questionnaire a exploréles données socioprofessionnelles, les habitudes de vie et les antécédents pathologiques familiaux et personnels de diabète, d'HTA, d'infarctus du myocarde, d'accident vasculaire cérébral, de dyslipidémie.

Les sujets étaient considérés comme fumeurs s'ils étaient fumeurs actuels ou en cours d'arrêt depuis moins d'un an. Pour la consommation d'alcool, on a comparé ceux qui consommaient de l'alcool contre ceux qui n'en avait jamais consommé. Le stress au travail a été évalué au moyen de la question «Selon vous, votre travail estil stressant ? ». Le régime alimentaire a été déterminé en demandant au salarié de décrire sa consommation journalière et en se référant à un outil utilisé par les praticiens de l'Association interprofessionnelle des centres médicaux de la région de l'Ile-de-France (12). Ainsi, la ration calorique qui correspond au petit-déjeuner, au déjeuner, au dîner ainsi qu'aux collations et grignotages a été calculée. Le régime alimentaire était réparti en trois classes, à savoir, petit mangeur (ration calorique quotidienne inférieure à $1599 \mathrm{kcal}$ ), mangeur moyen (ration calorique quotidienne entre 1600 et $2199 \mathrm{kcal}$ ) et gros mangeur (ration calorique quotidienne supérieure à $2200 \mathrm{kcal}$ ).

L'examen physique a comporté les mesures de la tension artérielle (TA), du poids, de la taille et du tour de taille (TT). La TA a été mesurée à l'aide d'un manomètre à capsule, en position assise, au repos au moins cinq minutes et en deux temps, au début et à la fin de l'entretien. La moyenne tensionnelle des deux mesures a été retenue. Le poids a été mesuré sur des sujets en tenue légère et déchaussés. La mesure de la taille a été pratiquée sur des sujets déchaussés, pieds à plat sur le sol, talons joints, dos contre le mur et la tête placée en position horizontale. Le TT a été mesuré sur des sujets dévêtus avec un mètre ruban non extensible, en prenant le diamètre minimal entre la crête iliaque et le bord costal inférieur. L'indice de masse corporelle (IMC) correspond au poids (en $\mathrm{kg}$ ) divisé par le carré de la taille $\left(\mathrm{en}^{2}\right.$ ). La classe de l'obésité a été déterminée en se référant à la classification de l'Organisation mondiale de la Santé (OMS) (13). Ainsi, le surpoids correspond à l'intervalle de l'IMC entre 25 et 29,9. L'obésité est définie par un IMC $\geq 30 \mathrm{~kg} / \mathrm{m}^{2}$.

Les prélèvements sanguins ont été effectués le matin, dans le même laboratoire d'analyses, en respectant toujours les mêmes conditions de prélèvement. Un jeune de 12 heures au minimum était préconisé pour le dosage de la glycémie, des triglycérides, du cholestérol HDL et du cholestérol total.

La définition établie par la Fédération internationale du diabète en 2005 a été adoptée pour le diagnostic du SM. Les critères retenus exigent comme critère obligatoire l'obésité abdominale (TT $\geq 94 \mathrm{~cm}$ chez l'homme ; $\geq 80 \mathrm{~cm}$ chez la femme) et deux autres critères, à savoir, pression artérielle systolique $\geq 130 \mathrm{mmHg}$ et/ou pression artérielle diastolique $\geq 85 \mathrm{mmHg}$ ou HTA traitée, glycémie à jeun $\geq 5,6 \mathrm{mmol} / \mathrm{L}$ ou diabète traité, triglycéridémie $\geq$ 1,7 mmol/L, cholestérol HDL<1,03 mmol/L chez l'homme et $<1,29 \mathrm{mmol} / \mathrm{L}$ chez la femme (14).

Les données ont été saisies et analysées grâce au logiciel SPSS 18.0. Les fréquences et les pourcentages ont été calculés pour les variables qualitatives. Les moyennes, les écarts types et l'étendue des valeurs extrêmes ont été calculés pour les variables quantitatives. Pour la comparaison des moyennes, le test t de Student a été utilisé. La comparaison des fréquences a été réalisée avec le test $\chi^{2}$ de Pearson. Pour l'étude multivariée, nous avons utilisé une régression logistique dont une variable dépendante, présence ou non de SM. Les variables indépendantes présentant une valeur de $p<0,250$ dans l'analyse univariée ont été incluses dans le modèle multivarié. Aussi, les variables indépendantes fortement corrélées n'ont pas été incluses dans le modèle. Pour tous les tests statistiques, le seuil de significativité $p$ a été fixé à 0,05.

\section{Résultats}

Au total, 263 agents de sexe masculin ont répondu à notre convocation, soit un taux de participation de $65,5 \%$. Le groupe des travailleurs postés représentait 48,3\% des participants, soit 127 agents, et le groupe des travailleurs non postés représentait 51,7 \%des participants, soit 136 agents. L'analyse des données socioprofessionnelles et des habitudes de vie des deux groupes est représentée dans le tableau 1.

La moyenne d'âge des travailleurs postés était de 43 (écart type [ET] 8,7) ans contre 41,6 (ET 10,1) ans pour les travailleurs non postés sans différence significative $(p=0,24)$. Le poste de technicien était le plus fréquemment occupé par les travailleurs postés et non postés chez 


\begin{tabular}{|c|c|c|c|c|c|}
\hline \multirow[t]{2}{*}{ Variables } & \multicolumn{2}{|c|}{ Travailleurs postés } & \multicolumn{2}{|c|}{ Travailleurs non postés } & \multirow[t]{2}{*}{$\mathbf{P}$} \\
\hline & Nombre & $\%$ & Nombre & $\%$ & \\
\hline \multicolumn{6}{|l|}{ Niveau d'études } \\
\hline Primaire & 8 & 7,4 & 10 & 6,3 & \multirow{3}{*}{0,55} \\
\hline Secondaire & 72 & 56,7 & 68 & 50 & \\
\hline Supérieur & 47 & 37 & 58 & 42,6 & \\
\hline \multicolumn{6}{|l|}{ Stuation matrimoniale } \\
\hline Marié & 93 & 73,2 & 91 & 66,9 & \multirow{2}{*}{0,26} \\
\hline Non marié & 34 & 26,8 & 45 & 33,1 & \\
\hline \multicolumn{6}{|l|}{ Nombre d'enfants à charge } \\
\hline$\leq 2$ & 55 & 59,1 & 54 & 57,4 & \multirow{2}{*}{0,81} \\
\hline$>2$ & 38 & 40,9 & 40 & 42,6 & \\
\hline \multicolumn{6}{|l|}{ Moyen de transport } \\
\hline Voiture & 61 & 48,4 & 65 & 51,6 & \multirow{2}{*}{0,75} \\
\hline Transport en commun & 61 & 44,9 & 75 & 55,2 & \\
\hline \multicolumn{6}{|l|}{ Tabagisme } \\
\hline Oui & 67 & 54,5 & 83 & 61,5 & \multirow{2}{*}{0,25} \\
\hline Non & 56 & 45,5 & 52 & 38,5 & \\
\hline \multicolumn{6}{|l|}{ Consommation d'alcool } \\
\hline Oui & 13 & 10,2 & 16 & 11,8 & \multirow{2}{*}{0,69} \\
\hline Non & 114 & 89,8 & 120 & 88,2 & \\
\hline \multicolumn{6}{|l|}{ Activité physique } \\
\hline Oui & 39 & 30,7 & 51 & 37,5 & \multirow{2}{*}{0,24} \\
\hline Non & 88 & 69,3 & 85 & 62,5 & \\
\hline \multicolumn{6}{|l|}{ Activité de loisir } \\
\hline Oui & 37 & 29,1 & 36 & 27,5 & \multirow{2}{*}{0,2} \\
\hline Non & 90 & 70,9 & 100 & 73,5 & \\
\hline \multicolumn{6}{|l|}{ Régime alimentaire } \\
\hline Petit mangeur & 6 & 4,7 & 4 & 2,6 & \multirow{3}{*}{0,71} \\
\hline Mangeur moyen & 61 & 48 & 76 & 50,3 & \\
\hline Gros mangeur & 60 & 47,2 & 71 & 47,0 & \\
\hline \multicolumn{6}{|l|}{ Poste de travail } \\
\hline Technicien & 73 & 57,5 & 54 & 39,7 & \multirow{4}{*}{0,06} \\
\hline Ouvrier & 31 & 24,4 & 42 & 32,5 & \\
\hline Ingénieur & 14 & 11 & 21 & 15,4 & \\
\hline Agent administratif & 9 & 7,1 & 17 & 12,5 & \\
\hline \multicolumn{6}{|l|}{ Exposition au bruit } \\
\hline Oui & 107 & 84,3 & 100 & 73,5 & 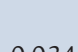 \\
\hline Non & 20 & 15,7 & 36 & 26,5 & \\
\hline Notion de stress & & & & & \\
\hline Oui & 67 & 52,8 & 73 & 53,7 & 000 \\
\hline Non & 60 & 47,2 & 63 & 46,3 & 0,80 \\
\hline
\end{tabular}


respectivement $57,5 \%$ et $39,7 \%$ des participants sans différence significative $(p=0,06)$. L'ancienneté professionnelle moyenne des travailleurs postés était de 14,1 (ET 9,4) ans contre 13,1 (ET 10,8) ans pour les travailleurs non postés sans différence significative $(p=0,43)$. Le nombre de cigarettes fumées par jour chez les travailleurs non postés était de 16,5 (ET 10) cigarettes contre 14,5 (ET $7,5)$ cigarettes chez les travailleurs postés sans différence significative $(p=0,16)$. La consommation alcoolique a concerné davantage le groupe des travailleurs non postés $(11,8 \%$ contre $10,2 \%)$ sans différence statistiquement significative $(p=0,69)$. L'activité sportive intéressait 30,7 \% des travailleurs postés contre $37,5 \%$ des travailleurs non postés, sans différence statistiquement significative $(p=$ 0,24). La ration calorique moyenne était de 2161 (ET 385) kcal pour les travailleurs postés contre 2164,3 (ET 345,8 ) kcal pour les travailleurs non postés sans différence significative $(p=0,94)$. Dans le groupe des travailleurs postés, le diabète était l'antécédent personnel le plus fréquent $(7,9 \%)$ et l'HTA l'était dans le groupe des trava illeurs non postés $(8,1 \%)$ sans différence statistiquement significative $(p=0,37)$. L'obésité était notée chez $43,3 \%$ des travailleurs postés et chez $39,7 \%$ des travailleurs non postés sans différence statistiquement significative $(p=0,81)$. Le tableau 2 représente la répartition des travailleurs en fonction des paramètres clinico-biologiques.

L'examen clinique et les mesures biologiques ont permis de retenir le diagnostic de SM chez 51,2\% des travailleurs postés et chez $27,2 \%$ des travailleurs non postés, avec une différence statistiquement significative $\left(p<10^{-3}\right)$.

$\mathrm{Au}$ terme de l'analyse univariée, les variables associées à la présence du SM étaient l'âge $\left(p<10^{-3}\right)$, la situation matrimoniale (marié) $\left(p<10^{-3}\right)$, le nombre d'enfants à charge $\left(p<10^{-3}\right)$, le régime alimentaire (gros mangeur) $\left(p<10^{-3}\right)$, les antécédents familiaux d'HTA $(p=0,03)$ et l'ancienneté professionnelle $\left(p<10^{-3}\right)$. L'activité sportive représentait un facteur protecteur contre le développement du SM ( $p=0,008)$ (Tableau 3). Après régression logistique binaire multiple, incluant les variables d'interêt, le travail posté semblait être associé au $\operatorname{SM}(p=0,003)$, avec un odds ratio (OR) de 2,64 (intervalle de confiance à $95 \%$ [IC $95 \%$ ] : 1,38-5,04) (Tableau 4).

\section{Discussion}

Au terme de cette étude, le SM était plus fréquent chez les travailleurs postés, avec une différence statistiquement significative $\left(p<10^{-3}\right)$. Cette association a persisté après ajustement des facteurs de confusion ( $p=0,003$; OR : 2,64 [1,38-5,04]).

Dans la littérature, plusieurs études ont trouvé des résultats similaires aux nôtres (15-18). Selon une étude japonaise, la prévalence du SM était plus importante chez les travailleurs postés que chez les travailleurs de jour. Les auteurs ont également rapporté une association

Tableau 2 Répartition des travailleurs en fonction des paramètres clinico-biologiques

\begin{tabular}{|c|c|c|c|c|c|}
\hline \multirow[t]{2}{*}{ Variables } & \multicolumn{2}{|c|}{ Travailleurs postés } & \multicolumn{2}{|c|}{ Travailleurs non postés } & \multirow[t]{2}{*}{$\mathbf{P}$} \\
\hline & Nombre & $\%$ & Nombre & $\%$ & \\
\hline \multicolumn{6}{|c|}{ Antécédents familiaux } \\
\hline Absents & 30 & 23,6 & 33 & 24,3 & \multirow{4}{*}{0,7} \\
\hline Diabète & 74 & 58,3 & 80 & 58,8 & \\
\hline HTA & 23 & 18,1 & 22 & 16,2 & \\
\hline Autres & 10 & 7,9 & 8 & 5,9 & \\
\hline \multicolumn{6}{|c|}{ Antécédents personnels } \\
\hline Absents & 92 & 72,4 & 107 & 78,7 & \multirow{4}{*}{0,37} \\
\hline Diabète & 10 & 7,9 & 7 & 5,1 & \\
\hline HTA & 8 & 6,3 & 11 & 8,1 & \\
\hline \multirow[t]{2}{*}{ Autres } & 17 & 13,4 & 11 & 8,1 & \\
\hline & \multicolumn{2}{|c|}{ Moyenne(ET) } & \multicolumn{2}{|c|}{ Moyenne (ET) } & \\
\hline IMC & \multicolumn{2}{|c|}{$29,3(3,9) \mathrm{kg} / \mathrm{m} 2$} & \multicolumn{2}{|c|}{$29,1(3) \mathrm{kg} / \mathrm{m} 2$} & 0,6 \\
\hline Tour de taille & \multicolumn{2}{|c|}{$98,4(9) \mathrm{cm}$} & \multicolumn{2}{|c|}{$94,8(8,2) \mathrm{cm}$} & 0,01 \\
\hline PAS & \multicolumn{2}{|c|}{$120(89) \mathrm{mmHg}$} & \multicolumn{2}{|c|}{$122(96) \mathrm{mmHg}$} & 0,67 \\
\hline $\mathrm{PAD}$ & \multicolumn{2}{|c|}{79 (37) $\mathrm{mmHg}$} & \multicolumn{2}{|c|}{79 (47) mmHg } & 0,82 \\
\hline Glycémie à jeun & \multicolumn{2}{|c|}{$5,6(1,4) \mathrm{mmol} / \mathrm{L}$} & \multicolumn{2}{|c|}{$5,4(1,2) \mathrm{mmol} / \mathrm{L}$} & 0,22 \\
\hline \multicolumn{6}{|l|}{ Profil lipidique } \\
\hline Triglycérides & \multicolumn{2}{|c|}{$1,4(0,5) \mathrm{mmol} / \mathrm{L}$} & \multicolumn{2}{|c|}{$1,2(0,5) \mathrm{mmol} / \mathrm{L}$} & 0,011 \\
\hline Cholestérol total & \multicolumn{2}{|c|}{$4,9(1,7) \mathrm{mmol} / \mathrm{L}$} & \multicolumn{2}{|c|}{$4,4(1) \mathrm{mmol} / \mathrm{L}$} & 0,04 \\
\hline Cholestérol HDL & \multicolumn{2}{|c|}{$0,41(0,15)$} & \multicolumn{2}{|c|}{$0,43(0,09)$} & 0,15 \\
\hline
\end{tabular}

HTA : hypertension artérielle; ET : écart type; IMC : indice de masse corporelle; PAS : pression artérielle systolique; PAD : pression artérielle diastolique. 


\begin{tabular}{|c|c|c|c|c|c|}
\hline \multirow[t]{3}{*}{ Variables } & \multicolumn{4}{|c|}{ Syndrome métabolique } & \multirow[t]{3}{*}{$p$} \\
\hline & \multirow[b]{2}{*}{ Nombre } & Oui & \multicolumn{2}{|c|}{ Non } & \\
\hline & & $\%$ & Nombre & $\%$ & \\
\hline \multicolumn{6}{|l|}{ Tranche d'âge (ans) } \\
\hline$<40$ & 24 & 23,5 & 74 & 46 & \multirow{2}{*}{$<10^{-3}$} \\
\hline$\geq 40$ & 78 & 76,5 & 87 & 54 & \\
\hline Situation matrimoniale (marié) & 89 & 84 & 108 & 62,8 & \\
\hline \multicolumn{6}{|l|}{ Tabagisme } \\
\hline Oui & 54 & 51 & 96 & 55,8 & \multirow{2}{*}{$<10^{-3}$} \\
\hline Non & 52 & 49 & 76 & 44,2 & \\
\hline \multicolumn{6}{|l|}{ Consommation d'alcool } \\
\hline Oui & 9 & 8,5 & 20 & 11,6 & \multirow{2}{*}{0,35} \\
\hline Non & 97 & 91,5 & 152 & 88,4 & \\
\hline \multicolumn{6}{|l|}{ Activité sportive } \\
\hline Oui & 26 & 24,5 & 69 & 40,1 & \multirow{2}{*}{0,4} \\
\hline Non & 80 & 75,5 & 103 & 59,9 & \\
\hline \multicolumn{6}{|l|}{ Régime alimentaire } \\
\hline Petit mangeur/mangeur moyen & 63 & 59,4 & 78 & 45,3 & \multirow{2}{*}{0,008} \\
\hline Gros mangeur & 43 & 40,6 & 94 & 54,7 & \\
\hline \multicolumn{6}{|l|}{ Notion de stress } \\
\hline Oui & 65 & 61,3 & 86 & 50 & \multirow{2}{*}{$<10^{-3}$} \\
\hline Non & 41 & 38,7 & 86 & 50 & \\
\hline \multicolumn{6}{|l|}{ Exposition au bruit } \\
\hline Oui & 78 & 73,5 & 73 & 42,4 & \multirow{2}{*}{0,06} \\
\hline Non & 28 & 38,6 & 99 & 57,5 & \\
\hline Antécédents familiaux d'HTA & 55 & 43,7 & 47 & 31,7 & 0,03 \\
\hline Antécédents familiaux de diabète & 44 & 42,1 & 56 & 32,5 & 0,1 \\
\hline Antécédents familiaux de dyslipidémie & 70 & 66,7 & 64 & 37,2 & 0,07 \\
\hline Antécédents familiaux d'IDM/AVC & 60 & 57,1 & 64 & 37,6 & 0,5 \\
\hline \multicolumn{6}{|l|}{ Type de travail } \\
\hline Travail non posté & 37 & 36,3 & 99 & 61,5 & \multirow{2}{*}{$<10^{-3}$} \\
\hline \multirow[t]{2}{*}{ Travail posté } & 65 & 63,7 & 62 & 38,5 & \\
\hline & \multicolumn{2}{|c|}{ Moyenne (ET) } & \multicolumn{2}{|c|}{ Moyenne (ET) } & \\
\hline Âge & \multicolumn{2}{|c|}{$45,9(8,7)$ ans } & & & $\leq 10^{-3}$ \\
\hline Ancienneté professionnelle & & & & & $\leq 10^{-3}$ \\
\hline Nombre d'enfants à charge & & & & & $\leq 10^{-3}$ \\
\hline
\end{tabular}

HTA: hypertension artérielle; IDM : infarctus du myocarde; $A V C$ : accident vasculaire cérébral; ET : écart type.

statistiquement significative entre le travail posté et le SM avec un risque de 6,30 (IC 95 \% : 1,24-32,15) (16). Une autre étude menée en Italie avait mis en évidence ce lien de causalité. Les auteurs avaient rapporté une incidence cumulée du SM de $9 \%$ chez les travailleurs de nuit et de 1,8\% chez les travailleurs de jour, et une incidence annuelle de 2,9\% et 0,5\% respectivement (17). Selon une étude belge, l'incidence du SM était significativement plus élevée chez les travailleurs postés. L'ajustement des facteurs de confusion a affecté 


\begin{tabular}{|c|c|c|c|}
\hline Variables & $\mathbf{O R}^{\mathbf{a}}$ & IC $95 \%$ & $\mathbf{P}$ \\
\hline Travail posté & 2,64 & $1,38-5,04$ & 0,003 \\
\hline Âge ( $\geq 40$ ans) & 1,1 & $0,4-3$ & 0,84 \\
\hline État civil (marié) & 1,6 & $0,1-19,5$ & 0,7 \\
\hline Nombre d'enfants (> 2) & 2,9 & $1,4-5,8$ & 0,002 \\
\hline Activité sportive & 0,51 & $0,25-1,04$ & 0,046 \\
\hline Régime alimentaire (gros mangeur) & 2,2 & $1,16-4,2$ & 0,015 \\
\hline Ancienneté professionnelle ( $\geq 10$ ans) & 0,81 & $0,39-1,66$ & 0,56 \\
\hline Notion de stress & 2,38 & $1,2-4,7$ & 0,013 \\
\hline Antécédents familiaux (diabète ou HTA ou dyslipidémie) & 2,68 & $1,2-5,9$ & 0,016 \\
\hline
\end{tabular}

uniquement le degré de l'association de façon marginale. Les résultats de cette étude suggèrent également une relation dose-réponse entre l'accumulation des années de travail posté et le développement du SM (18). Cependant, d'autres études ont rapporté des résultats différents. En effet, selon une étude japonaise, la prévalence du SM chez les travailleurs postés était significativement plus faible que celle chez les travailleurs de jour (10,7 \% contre 13,8 \%). Les auteurs n'avaient pas rapporté d'explications claires en raison du manque d'informations liées au travail et du faible effectif des travailleurs postés (19).

La désynchronisation des rythmes biologiques engendrée par les horaires atypiques du travail posté représente l'un des mécanismes physiopathologiques les plus avancés. Cette désynchronisation constitue le facteur initiateur de la perturbation de l'axe hypothalamohypophysaire avec un dérèglement de la température interne et des sécrétions hormonales d'une part, et de la perturbation du système nerveux central d'autre part (20).

Dans notre étude, après régression logistique multivariée, les autres variables associées à la présence du SM étaient le nombre d'enfants, le régime alimentaire (gros mangeur) et la notion de stress au travail. L'activité sportive était un facteur protecteur contre le développement du SM.

Plusieurs études ont tenté d'analyser les variables associées à la survenue du SM. Il est bien établi que la prévalence du SM dépend fortement de l'âge. Cette constatation pourrait être expliquée par la variation de toutes les composantes du SM avec l'âge (3). Dans notre étude, les salariés ayant un SM étaient plus âgés que ceux indemnes, sans différence statistiquement significative. Ceci peut être expliqué par la prédominance des travailleurs assez jeunes qui ont participé à cette étude. Cependant, il faut signaler que le SM touche actuellement de plus en plus les sujets jeunes, y compris les enfants, en raison du changement dans les habitudes alimentaires, de la sédentarité et de l'augmentation de la prévalence de l'obésité dans cette catégorie (21).

Quant à l'effet du sexe, il n'existe pas de conclusions définitives du fait de la grande discordance des résultats avancés sur ce sujet (22). Dans notre étude, ce paramètre n'a pas été étudié puisque seuls les hommes ont été inclus. Cependant, les résultats d'une étude tunisienne suggèrent une prédominance féminine du SM (23) et rejoignent ceux des pays du Moyen-Orient et d'Afrique du Nord (24). Cette prédominance féminine peut être due à une plus grande prévalence de l'obésité abdominale et de la sédentarité chez les femmes orientales mais aussi aux différences ethniques du TT (23). Contrairement à ces constatations, le SM était plus fréquent chez les hommes dans les pays occidentaux (3).

Dans notre étude, le nombre d'enfants à charge était significativement associé au risque de développer le SM. Le stress lié à la gestion d'une telle charge sociale pourrait expliquer en partie cette association. En effet, certaines études ont rapporté une association entre le stress chronique et les maladies métaboliques, notamment le SM (25). Cette constatation a été également rapportée dans notre enquête.

Le poste de travail ainsi que l'ancienneté profession nelle ne semblaient pas influencer la survenue du SM dans notre étude. Selon une étude menée au Pérou, la prévalence du SM était de $28,9 \%$ chez les travailleurs administratifs et de $23 \%$ chez les travailleurs en zone industrielle. Les auteurs expliquent ce résultat par le régime hypercalorique et la sédentarité chez les travailleurs de bureau (26). Il a été aussi démontré qu'il existe une augmentation de la prévalence du SM chez des travailleurs ayant dépassé une ancienneté de plus de 10 ans (18).

On dispose actuellement de plusieursrésultats émanant des études ayant démontré le rôle protecteur de l'exercice physique contre le développement du SM, ce qui concorde avec nos résultats. En effet, une activité physique à raison de trois séances de 20 minutes par semaine pendant 20 semaines pourrait diminuer de 16,9\% la prévalence du SM, de $43 \%$ le taux des triglycérides et de $9 \%$ la glycémie (27).

Comme rapporté dans notre étude, les travailleurs classés comme étant de gros mangeurs étaient plus vulnérables au développement d'un SM. Selon Basdevant (28), cette augmentation des apports alimentaires pourrait être expliquée par les troubles du comportement alimentaire, la convivialité, les habitudes familiales et les sollicitations professionnelles. Esquirol et al., en comparant le régime alimentaire des travailleurs 
postés et non postés, avaient conclu que l'apport énergétique des travailleurs postés au cours de la journée était plus fractionné et qu'il était beaucoup plus élevé pendant les repas intermédiaires (15).

Les données sur la relation entre le tabagisme et le risque de SM sont contradictoires. Aucune association n'a été observée aussi bien dans notre étude que dans celle de Bhanushali et al. (29). Cependant, Akbartabartoori et al. ont rapporté que les fumeurs avaient un IMC plus bas que les non-fumeurs mais un TT plus élevé, suggérant une augmentation de la graisse abdominale et une diminution de la sensibilité à l'insuline (30).

Dans notre étude, nous n'avons pas trouvé d'association entre la consommation d'alcool et le risque de SM. Ce constat était concordant avec les résultats de l'enquête française EPIMIL (31). En revanche, Fan et al. ont trouvé que la consommation excessive d'alcool entraîne une augmentation des triglycérides et de la tension artérielle, en plus d'un apport énergétique pouvant conduire à un gain de poids (32).

Bien que notre étude se veuille exhaustive, certaines limites méritent d'être signalées. En effet, le taux de participation était de $65,5 \%$. Ce taux peut être expliqué par la durée relativement prolongée de l'entretien, puisque le recueil des données par le questionnaire et l'examen physique a nécessité en moyenne une demi-heure par personne. D'autre part, bien que la nature transversale de l'étude ait l'avantage d'être plus pratique, de faible coût et de courte durée, elle ne permet pas cependant d'établir de liens de causalité entre les variables étudiées. En plus, l'utilisation d'un auto-questionnaire dans le recueil des données présente un biais de subjectivité pour certains paramètres, notamment ceux en rapport avec le stress et le bruit. Finalement, la diversité des définitions utilisées aussi bien pour le SM que pour le travail posté ne permet pas de bien cerner la problématique et de tirer des conclusions définitives. Cependant, et malgré ces limites, notre population a l'avantage d'être homogène. En effet, les travailleurs appartenant à la même entreprise subissent les mêmes contraintes. De même, l'examen clinique a été réalisé par le même médecin afin de réduire le biais de mesure. Finalement, la liste des variables étudiées était assez exhaustive et a cadré avec l'ensemble des facteurs de risque potentiels impliqués dans la liaison de la chaîne causale complexe du travail posté et du SM.

\section{Conclusion}

Le développement économique et industriel au cours des dernières décennies a accru de façon spectaculaire le nombre de travailleurs en horaires atypiques. Ce mode de travail est responsable du dérèglement du fonctionnement des rythmes biologiques et de lourdes conséquences sur l'opérateur pouvant s'exprimer par diverses pathologies, notamment le syndrome métabolique, comme le souligne notre étude. Ainsi, l'application de mesures préventives s'avère nécessaire afin de protéger les salariés. Cette prévention repose non seulement sur des mesures hygiéno-diététiques et une activité physique régulière mais aussi sur l'amélioration des conditions de travail.

Financement : aucun.

Conflits d'intérêts : aucun déclaré.

\section{Evaluation of the risk of metabolic syndrome among shift workers in Tunisia}

\section{Abstract}

Background: Shift work is a source of health problems for workers around the world. The metabolic syndrome is one of the pathologies that can occur because of this work pattern.

Aims: Investigate possible association between shift work and the development risk of the metabolic syndrome (MS).

Methods: A cross-sectional study was conducted among male gender agents of company of electricity production in the Center of Tunisia. This population was divided into two groups according to the organization of their work: a group of shift workers and a group of non-shift workers. Data collection was based on a questionnaire, a clinical examination and a biological assessment. The definition of International Diabetes Federation 2005 (IDF) was adopted to retain the diagnosis of the MS.

Results: A total of 263 agents responded to our invitation with a participation rate of $65.5 \%$. The shift workers group accounted for $48.3 \%$ of the participants and the fixed workers group accounted for $51.7 \%$ of the participants. The diagnosis of MS was retained in $51.2 \%$ of shift workers and $27.2 \%$ of non-posted workers, with a statistically significant difference ( $p$ $<10-3)$. This association persisted after adjusting for the confounding variables with an OR of 2.64 [1.38-5.04].

Conclusion: Our study underlined a higher risk of MS development among shift workers. These findings highlight the importance of taking specific preventive measures. This prevention is based not only on hygieno-dietetic measures and regular physical activity, but also the improvement of working conditions. 


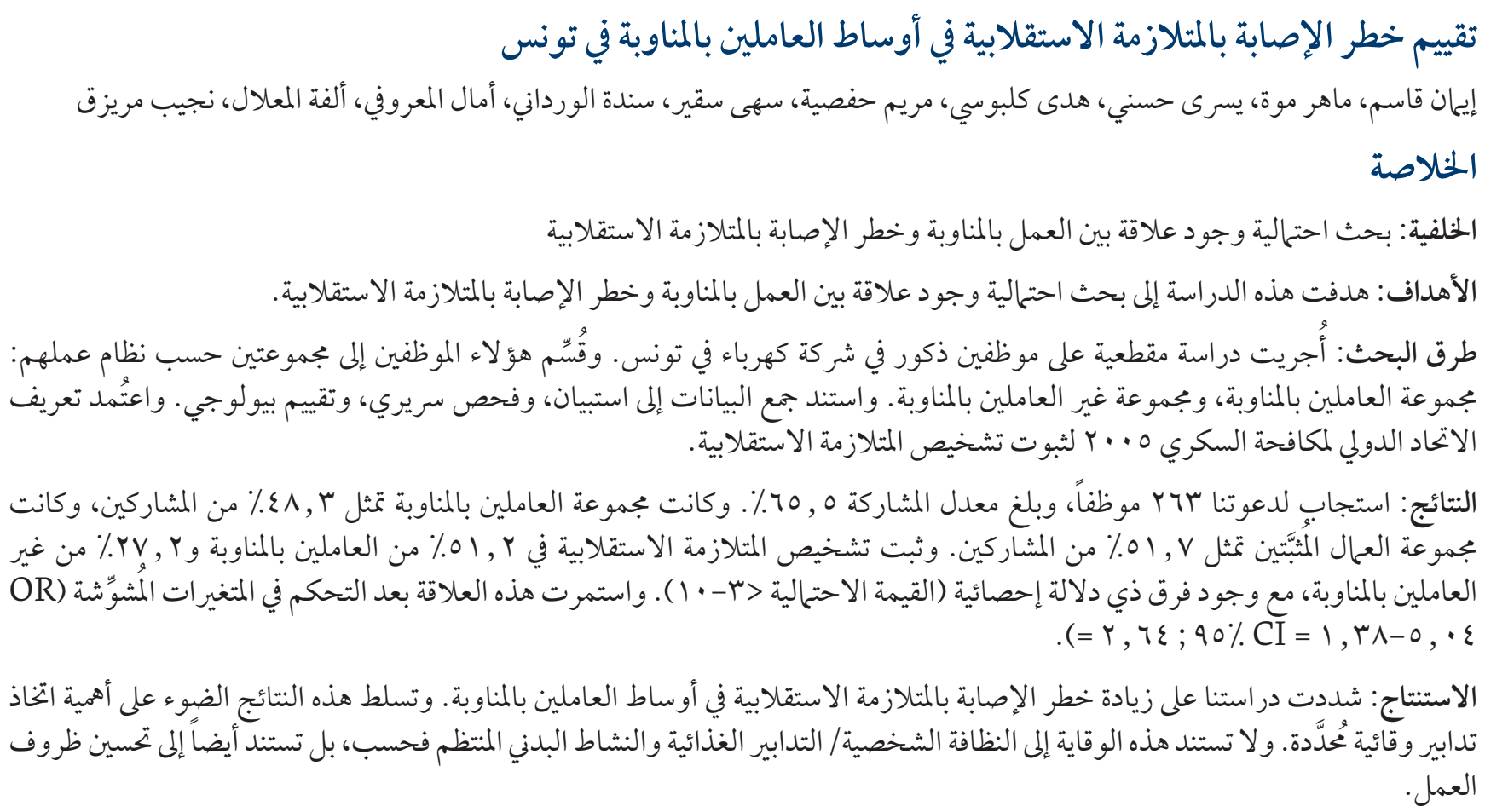

\section{Références}

1. Eckel RH, Grundy SM, Zimmet PZ. The metabolic syndrome. Lancet. 2005 Apr 16-22;365(9468):1415-28. https://doi. org/10.1016/S0140 - 6736(05)66378 - 7 PMID:15836891

2. Wu SH, Hui WS, Liu Z, Ho SC. Metabolic syndrome and all - cause mortality: a meta - analysis of prospective cohort studies. Eur J Epidemiol. 2010 Jun;25(6):375-84. https://doi.org/10.1007/s10654-010-9459 - z PMID:20425137

3. Ford ES, Giles WH, Mokdad AH. Increasing Prevalence of the Metabolic Syndrome among U.S. Adults. Diabetes Care. 2004 Oct;27(10):2444-9. PMID:15451914

4. Allal-Elasmi M, Haj Taieb S, Hsairi M, Zayani Y, Omar S, Sanhaji H, et al. The metabolic syndrome: prevalence, main characteristics and association with socio - economic status in adults living in Great Tunis. Diabetes Metab. 2010 Jun;36(3):204-8. https://doi.org/10.1016/j.diabet.2009.11.009 PMID:20202880

5. Kesse-Guyot E, Ahluwalia N, Lassale C, Hercberg S, Fezeu L, Lairon D. Adherence to Mediterranean diet reduces the risk of metabolic syndrome: A 6 - year prospective study. Nutr Metab Cardiovasc Dis. 2013 Jul;23(7):677-83. https://doi.org/10.1016/j. numecd.2012.02.005 PMID: 22633793

6. Canuto R, Pattussi MP, Macagnan JBA, Henn RL, Olinto MTA. Metabolic syndrome in fixed - shift workers. Rev. Saúde Públ. 2015;49:30. http://dx.doi.org/10.1590/S0034 - 8910.2015049005524 PMID:26061455

7. Gaudemaris RD, Lang T. Organisation et contraintes du travail : un nouveau facteur de risque cardiovasculaire. Arch Mal Prof Enviro. 2002;63(3 - 4):320-5. https://doi.org/ADMP - 06 - 2002 - 63 - 3 - 1250 - 3274 - 101019 - ART273

8. Morris CJ, Aeschbach D, Scheer FA. Circadian system, sleep and endocrinology. Mol Cell Endocrinol. 2012 Feb;349(1):91-104. https://doi.org/10.1016/j.mce.2011.09.003 PMID:21939733

9. Puttonen S, Härmä M, Hublin C. Shift work and cardiovascular disease - pathways from circadian stress to morbidity. Scand J Work Environ Health. 2010 Mar;36(2): 96-108. PMID:20087536

10. Karlsson B, Knutsson A, Lindahl B. Is there an association between shift work and having a metabolic syndrome? Results from a population based study of 27,485 people. Occup Environ Med. 2001 Nov;58(11):747-52. PMID:11600731

11. Canuto R, Garcez AS, Olinto MTA. Metabolic syndrome and shift work: a systematic review. Sleep Med Rev. 2013 Dec;17(6):425-31. https://doi.org/10.1016/j.smrv.2012.10.004 PMID: 23531362

12. Alcouffe J, Bourasset D, Wernicki - Morand A, Paupin F. Faut - il crier haro sur les gros ? Concours Med. 1999 Avril;121(15):1151-5.

13. Obésité [site web]. Organisation mondiale de la Santé. (https://www.who.int/topics/obesity/fr/, consulté le 28 mars 2019 ).

14. Alberti KG, Zimmet P, Shaw J; IDF Epidemiology Task Force Consensus Group. The metabolic syndrome - - a new worldwide definition. Lancet. 2005;366(9491):1059-62. https://doi.org/10.1016/S0140 - 6736(05)67402 - 8 PMID:16182882.

15. Esquirol Y, Bongard V, Mabile L, Jonnier B, Soulat JM, Perret B. Shift work and metabolic syndrome: respective impacts of job strain, physical activity, and dietary rhythms. Chronobiol Int. 2009 Apr;26(3):544-59. https://doi. org/10.1080/07420520902821176 PMID:19360495 
16. Ye HH, Jeong JU, Jeon MJ, Sakong J. The Association between Shift Work and the Metabolic Syndrome in Female Workers. Ann Occup Environ Med. 2013;25(1):33. https://doi.org/10.1186/2052 - 4374-25 - 33 PMID:24472469

17. Pietroiusti A, Neri A, Somma G, Coppeta L, Iavicoli I, Bergamaschi A, et al. Incidence of metabolic syndrome among night- shift healthcare workers. Occup Environ Med. 2010 Jan;67(1):54-7. https://doi.org/10.1136/oem.2009.046797 PMID:19737731

18. De Bacquer D, Van Risseghem M, Clays E, Kittel F, De Backer G, Braeckman L. Rotating shift work and the metabolic syndrome: a prospective study. Int J Epidemiol. 2009 Jun;38(3):848-54. https://doi.org/10.1093/ije/dyn360 PMID:19129266

19. Kawada T, Otsuka T, Inagaki H, Wakayama Y, Katsumata M, Li Q, et al. A cross - sectional study on the shift work and metabolic syndrome in Japanese male workers. Aging Male. 2010 Sep;13(3):174-8. https://doi.org/10.3109/13685530903536692 PMID:20059304

20. Léger D, Bayon V, Metlaine A, Prevot E, Didier - Marsac C, Choudat D. Horloge biologique, sommeil et conséquences médicales du travail posté. Arch Mal Prof Environ. 2009 Juin;70(3):246-52. https://doi.org/10.1016/j.admp.2009.01.007

21. Lameira D, Lejeune S, Mourad JJ. Le syndrome métabolique : son épidémiologie et ses risques. Ann Dermatol Venereol. 2008 Feb;135(4):S249-53. https://doi.org/10.1016/S0151 - 9638(08)70543 - X PMID:18466792

22. Kolovou GD, Anagnostopoulou KK, Salpea KD, Mikhailidis DP. The prevalence of metabolic syndrome in various populations. Am J Med Sci. 2007 Jun;333(6):362-71. https://doi.org/10.1097/MAJ.0b013e318065c3al PMID:17570989

23. Bouguerra R, Ben Salem L, Alberti H, Ben Rayana C, El Atti J, Blouza S, et al. Prevalence of metabolic abnormalities in the Tunisian adults: a population based study. Diabetes Metab. 2006 Jun;32(3):215-21. PMID:16799397

24. Al-Lawati JA, Mohammed AJ, Al-Hinai HQ, Jousilahti P. Prevalence of the metabolic syndrome among Omani adults. Diabetes Care. 2003 Jun;26(6):1781-5. PMID:12766109

25. Magnavita N, Fileni A. Work stress and metabolic syndrome in radiologists: first evidence. Radiol Med. 2014 Feb;119(2):142-8. https://doi.org/10.1007/s11547-013-0329-0 PMID:24297580

26. Baldeon JCP, Chumbes GCN. Síndromemetabólico y puesto de trabajo [Metabolic syndrome and workplace]. Med Segur Trab. 2010 Dec;56(221):280-7. https://doi.org/10.4321/S0465 - 546X2010000400004 (http://scielo.isciii.es/pdf/mesetra/v56n221/ original2.pdf, consulté le 28 février 2019)

27. Katzmarzyk PT, Leon AS, Wilmore JH, Skinner JS, Rao DC, Rankinen T, et al. Targeting the metabolic syndrome with exercise: evidence from the HERITAGE Family Study. Med Sci Sports Exerc. 2003 Oct;35(10):1703-9. https://doi.org/10.1249/01. MSS.0000089337.73244.9B PMID:14523308

28. Basdevant A. Analyse clinique du comportement alimentaire. Rev Prat. 2000;50(5):484-8.

29. Bhanushali CJ, Kumar K, Wutoh AK, Karavatas S, Habib MJ, Daniel M, et al. Association between Lifestyle Factors and Metabolic Syndrome among African Americans in the United States. J Nutr Metab. 2013;2013:516475. https://doi. org/10.1155/2013/516475 PMID:23431427

30. Akbartabartoori M, Lean ME, Hankey CR. Relationships between cigarette smoking, body size and body shape. Int J Obes (Lond). 2005 Feb;29(2):236-43. https://doi.org/10.1038/sj.ijo.0802827 PMID:15505632

31. Bauduceau B, Baigts F, Bordier L, Burnat P, Ceppa F, Dumenil V, et al. Epidemiology of the metabolic syndrome in 2045 French military personnel (EPIMIL study). Diabetes Metab. 2005 Sep;31(4 Pt 1):353-9.PMID:16369197

32. Fan AZ, Russell M, Naimi T, Li Y, Liao Y, Jiles R, et al. Patterns of alcohol consumption and the metabolic syndrome. J Clin Endocrinol Metab. 2008 Oct;93(10):3833-8 .https://doi.org/10.1210/jc.2007-2788 PMID:18628524 